\title{
A Hipoglicemia Silenciosa é Parte do Controle Glicêmico Ideal em Pacientes com DM1? - Tempo de Hipoglicemia pelo CGMS versus Média Glicêmica
}

\section{original}

\author{
FrEDERICO FERNANDES RIBEIRO MAIA \\ LeVimar R. Araújo
}

Departamento de Fisiologia da

Faculdade de Ciências Médicas de Minas Gerais (FCMMG);

Clínica de Endocrinologia e Metabologia do Hospital Universitário São José da FCMMG, Belo Horizonte, MG, Brasil.

\section{RESUMO}

Objetivo: Avaliar o impacto do tempo de hipoglicemia silenciosa no controle glicêmico de pacientes diabéticos tipo 1 (DM1) sob monitorização contínua de glicose (CGMS). Métodos: Oitenta e sete pacientes DM1 $(45 \% \mathrm{M} / 55 \% \mathrm{~F})$, divididos em quatro grupos, submetidos à CGMS 72 horas. Foram analisados: hipoglicemia silenciosa (HS) $(<70 \mathrm{mg} / \mathrm{dL})$; tempo de hipoglicemia pelo CGMS, sendo os pacientes classificados em G1 (<5\%), G2 (5-10\%), G3 (10\% a $20 \%$ ) e G4 (> 20\%); níveis de A1c e médias glicêmicas. Resultados: A HS foi detectada em $64,5 \%$ dos casos, sendo mais duradoura (mín.) durante a noite versus o dia $(\mathrm{p}<0,001)$. Quanto ao tempo de HS, $41,4 \%$ dos pacientes ficaram $<5 \%, 21,8 \%$ entre $5 \%$ a $10 \%, 23 \%$ entre $10 \%$ a $20 \%$ e $13,8 \%$ com $>20 \%$ do CGMS 72 horas. Verificou-se menor média glicêmica quanto maior o tempo de hipoglicemia $(p=0,006)$. Conclusão: A hipoglicemia silenciosa é freqüente em pacientes com DM1, no período noturno. Observou-se tempo de $10 \%$ a $20 \%$ de hipoglicemia silenciosa para a média glicêmica entre 120 a $160 \mathrm{mg} / \mathrm{dL}$. (Arq Bras Endocrinol Metab 2008; 52/6:994-1000)

Descritores: Hipoglicemia silenciosa; A1c; Média glicêmica; Diabetes melito tipo 1

\section{ABSTRACT}

Is Silent Hypoglycemia Part of Ideal Glycemic Control in DM1 Patients? Hypoglycemic State by CGMS vs. Glycemic Average.

Background: To evaluate the impact of silent hypoglycemic state in glycemic control in type 1 diabetic patients (DM1) by CGMS. Methods: 87 DM1 patients $(45 \% \mathrm{M} / 55 \% \mathrm{~F})$ submitted to a $72 \mathrm{~h}$ CGMS profile were classified in 4 groups. It was analyzed: unrecognized hypoglycemia $(<70 \mathrm{mg} / \mathrm{dL})$; duration time of silent hypoglycemia in which patients were classified into G1 $(<5 \%)$, G2 $(5-10 \%)$, G3 $(10-20 \%)$ and G4 (>20\%) of hypoglycemic state by CGMS; A1c and mean capillary glucose (MCG) in each group. Results: The silent hypoglycemia was detected in $64.5 \%$ of patients and nighttime episodes of hypoglycemia lasted longer $(\mathrm{min})$ than daytime episodes in all groups $(p<0.001)$. It was verified $41.4 \%$ of patients under than $5 \%$ of time in hypoglycemic state, $21.8 \%$ between $5-10 \%, 23 \%$ between $10-20 \%$ and $13.8 \%$ with more than $20 \%$ of CGMS in silent hypoglycemia. This data showed significant decreased in MCG when the duration time of silent hypoglycemia was longer $(p=0.006)$. Conclusion: The silent hypoglycemia is common in DM1 patients and most frequently in night time period. To take an average glycemia of $120-160 \mathrm{mg} / \mathrm{dL}$ in these patients, it was necessary a $10-20 \%$ of CGMS period in silent hypoglycemia in these patients. (Arq Bras Endocrinol Metab 2008; 52/6:994-1000)

Recebido em 10/1/2008

Key-words: Silent hypolgycemia; A1c; Average glycemia; Diabetes mellitus type 1 


\section{INTRODUÇÃO}

A PESAR DO APARECIMENTO de novas drogas, novas técnicas de monitorização e melhor conhecimento da fisiopatologia do diabetes melito (DM), o controle metabólico permanece insatisfatório na maioria da população diabética. Dados americanos mostram que menos da metade $(<50 \%)$ dos diabéticos mantêm sua doença bem controlada e dados nacionais de 6.701 pacientes com DM (15\% DMl /85\% DM2) mostram Alc > 7\% em 90\% no DMl e 73\% no DM2 $(1,2)$.

A American Diabetes Association (ADA) recomenda glicohemoglobina $(\mathrm{Alc})<6,5 \%$, glicemia pré-prandial $(\mathrm{FPG})<110 \mathrm{mg} / \mathrm{dL}$ e glicemia pós-prandial $(\mathrm{PPG})$ $<140 \mathrm{mg} / \mathrm{dL}$, como alvos de controle satisfatório (1). Os grandes estudos, DCCT (Diabetes Control and Complications Trial) e UKPDS (United Kingdom Prospective Diabetes Study), mostraram que a redução de 1\% na $\mathrm{Alc}$ influi significativamente na proteção à microangiopatia e à neuropatia (3). No entanto, mesmo pacientes com níveis adequados de Alc experimentam episódios freqüentes de hipoglicemia noturna e hiperglicemia pós-prandial (HPP), possíveis complicadores da adesão ao tratamento intensivo e rígido $(3,4)$.

Estudos diversos evidenciam o impacto da monitorização contínua de glicose (CGMS) na melhora do controle metabólico, detecção de excursões glicêmicas, HPP e hipoglicemia silenciosa (60\% a 70\%), em pacientes com DM1, DM2, idosos, brittle diabetes e crianças, inclusive menores de 7 anos (5-13). Em pacientes com DMl, a hipoglicemia noturna é a complicação freqüente do tratamento intensivo, visto que $55 \%$ dos episódios graves ocorreram durante a noite no DCCT (3).
Desde as publicações dos dados do DCCT e do UKPDS, ficou estabelecida a alta correlação entre os níveis de glicohemoglobina (Alc) e as futuras complicações, especialmente as microvasculares (3). A Alc tem sido utilizada no meio médico como marcador de controle glicêmico em pacientes diabéticos. A medida avalia a proporção de hemoglobina glicada em sua porção $\mathrm{N}$-terminal da cadeia $\beta$ e reflete o controle glicêmico nos últimos três meses. Os dados de Rohlfing e cols. (14) estabeleceram a correlação entre a média glicêmica (glicemia capilar $7 \times /$ dia) com a Alc a partir dos dados de 1.439 pacientes do DCCT (Tabela 1).

No entanto, a correlação entre média glicêmica e Alc ainda é tema de discussão. Em virtude das variações dos métodos laboratoriais para medida da Alc (mais de 30 disponíveis) e das variações genéticas da hemoglobina, a padronização e a comparação dos resultados tornam-se difíceis. Uma vez que a Alc reflete a glicemia média dos últimos três meses, estima-se que menor nível de Alc/média glicêmica esteja associado a maior período de hipoglicemia nesses pacientes. Segundo revisão em MedLine/PubMed nos últimos dez anos, o impacto do tempo de hipoglicemia silenciosa sobre a média glicêmica e Alc em pacientes com DMl não é conhecido, o que motivou a realização deste estudo.

\section{CASUÍsticA E MÉTOdOS}

Foi realizado estudo retrospectivo, transversal, com análise qualitativa e quantitativa, de pacientes com DMl, atendidos na Clínica de Endocrinologia do Hospital Universitário São José (CEM-HUSJ), no período de julho de 2005 a julho de 2007.

Tabela 1. Estimativa de média glicêmica versus Alc em pacientes com DM1 pelo DCCT.

\begin{tabular}{|c|c|c|c|c|}
\hline \multirow[t]{2}{*}{$\mathrm{HbA}_{1 \mathrm{c}}(\%)$} & \multicolumn{2}{|c|}{ MPG estimada pela regressão } & \multicolumn{2}{|c|}{ MPG aproximada para uso clínico } \\
\hline & $\mathrm{mmol} / \mathrm{L}$ & $\mathrm{mg} / \mathrm{dL}$ & $\mathrm{mmol} / \mathrm{L}$ & $\mathrm{mg} / \mathrm{dL}$ \\
\hline 4 & 3.6 & 65 & 3.5 & 65 \\
\hline 5 & 5.6 & 101 & 5.5 & 100 \\
\hline 6 & 7.6 & 137 & 7.5 & 135 \\
\hline 7 & 9.6 & 172 & 9.5 & 170 \\
\hline 8 & 11.5 & 208 & 11.5 & 205 \\
\hline 9 & 13.5 & 244 & 13.5 & 240 \\
\hline 10 & 15.5 & 279 & 15.5 & 275 \\
\hline 11 & 17.5 & 315 & 17.5 & 310 \\
\hline 12 & 19.5 & 350 & 19.5 & 345 \\
\hline
\end{tabular}

MPG = média plasmática glicêmica; Fonte: Adaptado de Rohlfing CL e cols., Diabetes Care. 2002;25(2):275-8. 


\section{Pacientes}

Foram avaliados 87 pacientes com DMl, divididos em quatro grupos, de acordo com o tempo de hipoglicemia silenciosa ao CGMS 72 horas: Gl (<5\%), G2 $(5 \%$ a $10 \%), \mathrm{G} 3(10 \%$ a $20 \%)$ e G4 (> $20 \%)$; sendo $45 \%$ do sexo masculino e $55 \%$ do sexo feminino. A idade variou de 1 a 40 anos, com média de $21,8 \pm 11,5$ anos. Não houve restrição quanto a idade, sexo ou tempo de diabetes. O tempo de diabetes foi de $9,4 \pm 8,2$ anos.

\section{Sensor de glicose (CGMS)}

A CGMS foi realizada pelo monitor Medtronic/Northridge, CA. A glicose é mensurada com base na reação eletroquímica da enzima glicose oxidase presente no sensor com a glicose do fluido intersticial. Os valores variam entre 40 e $400 \mathrm{mg} / \mathrm{dL}$ e são captados a cada 10 segundos, com o registro da média desses valores a cada 5 minutos, no total de 288 medidas ao dia e 864 medidas durante o exame ( 72 horas), conforme recomendação do fabricante. Sessenta minutos após a instalação, a corrente elétrica em nanoamperes gerada é convertida em glicose, após a inclusão de um valor de glicemia capilar no monitor. Ao final do registro, o sensor é descartado e os dados armazenados são transferidos ao software, para análise e interpretação.

\section{MÉTODOS}

Todos os pacientes foram submetidos previamente às orientações básicas de funcionamento do monitor, pelo mesmo examinador, técnica de manuseio (registro de eventos e glicemia no monitor), cuidados gerais e preenchimento do "diário do paciente". Os pacientes foram orientados a realizar e incluir, no mínimo, quatro valores de GC no monitor por dia, com intervalo de cerca de 6 horas entre as medidas. A medida noturna da GC foi orientada a todos os participantes, dentro de sua rotina de vida. A primeira GC foi realizada após 60 minutos do início da monitorização contínua.

As variáveis foram: glicemia capilar média (GCM); glicose média pelo sensor CGMS; coeficiente de correlação (\%) entre GC e glicose pelo sensor; presença de hipoglicemia silenciosa $(<70 \mathrm{mg} / \mathrm{dL}$ ) (freqüência e duração), durante o dia e noite; tempo de hipoglicemia, classificado entre < $5 \%$ (G1), $5 \%$ a $10 \%(\mathrm{G} 2), 10 \%$ a $20 \%$ (G3) e $>20 \%$ (G4) do registro de 72 horas; e níveis de Alc e médias glicêmicas, de acordo com o tempo de hipoglicemia estudado em cada grupo.
As excursões glicêmicas identificadas pela GC foram fundamentadas na informação ("diário") do paciente e análise do glicosímetro e comparadas com as excursões da glicose detectadas pelo sensor. Consideraram-se excursões glicêmicas valores de glicemia $<70 \mathrm{mg} / \mathrm{dL}$ (hipoglicemia) e $>140 \mathrm{mg} / \mathrm{dL}$ (hiperglicemia).

A presença de HPP foi definida para valores de glicemia superiores a $140 \mathrm{mg} / \mathrm{dL}$ duas horas após o almoço. A hipoglicemia noturna silenciosa foi definida por glicemia $<70 \mathrm{mg} / \mathrm{dL}$ no período entre 23 e 7 horas, sendo registrado em minutos durante o dia versus a noite, para efeito de comparação.

Os níveis de glicohemoglobina (Alc) foram determinados em cada grupo (Gl a G4), na semana de uso do CGMS. Os valores de Alc foram estabelecidos pelo método HPLC (cromatografia líquida de alta performance), com referência entre $4,3 \%$ e $6,9 \%$. A média glicêmica pelo CGMS foi estratificada em cada grupo, comparativamente à Alc.

\section{Estatística}

Os dados foram coletados em ficha-padrão e analisados pelo software Minitab, versão 14, por meio do teste qui-quadrado e teste $t$ de Student. Os dados são apresentados como média/desvio-padrão. As variáveis foram comparadas entre os quatro grupos, considerando um $\mathrm{p}<0,05$ para significância estatística.

\section{RESULTADOS}

O número de leituras do sensor foi $>90 \%$ sem diferença significante. A GC média foi de $161,3 \pm 60,5 \mathrm{mg} / \mathrm{dL}$ versus $163,2 \pm 64,8 \mathrm{mg} / \mathrm{dL}$ detectada pelo sensor, sem diferença significante $(\mathrm{p}=0,7 \mathrm{l})$. O CGMS detectou mais excursões glicêmicas em relação à $\mathrm{GC}$ média $(12,1$ $\pm 6,3$ versus $8,4 \pm 4,9 ; \mathrm{p}<0,001)$.

A HPP foi evidenciada em $57,1 \%$ dos casos, com valor médio de $140,6 \pm 85,2 \mathrm{mg} / \mathrm{dL}(\mathrm{VR}<140 \mathrm{mg} / \mathrm{dL})$. A hipoglicemia silenciosa foi detectada em $64,5 \%$ dos casos. A hipoglicemia silenciosa foi estatisticamente mais duradoura (mín.) durante o período noturno em relação ao diurno $(189,5 \pm 29,5$ versus $80,3 \pm 19,6 ; \mathrm{p}<0,001)$.

Quanto ao tempo de hipoglicemia (em 72 horas de CGMS), verificou-se que quase metade dos pacientes $(41,4 \%)$ permaneceu por menos de $5 \%$ do registro da CGM e cerca de um quarto dos pacientes com duração de $10 \%$ a $20 \%$ do tempo em hipoglicemia silenciosa (Figura 1). 


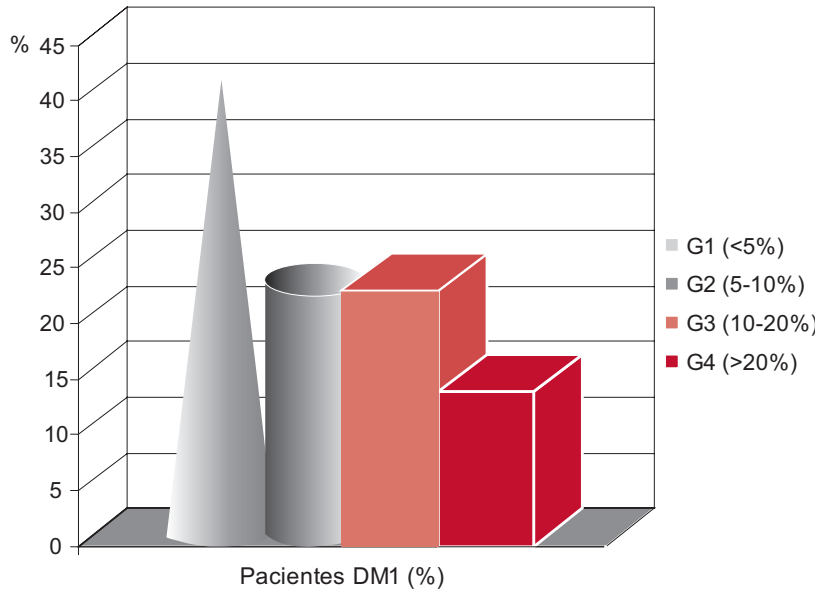

Figura 1. Classificação dos pacientes com DM1 pelo tempo de hipoglicemia silenciosa (\%) pelo CGMS 72 horas.

Observou-se menor média glicêmica quanto maior o tempo de hipoglicemia, com valor significante $(\mathrm{p}=$ $0,006)$, o que pode ser corroborado pelos valores de Alc de maneira semelhante (Tabela 2 ), não verificado no G4. $\mathrm{A}$ presença de $\mathrm{Alc}<7 \%$ esteve associada à média glicêmica de 168,69 mg/dL (variação: 108 a $264 \mathrm{mg} / \mathrm{dL}$ ).

Quanto ao tempo de hiperglicemia durante o dia, verificou-se que os pacientes permaneceram em média glicêmica elevada em quase $50 \%$ do tempo de registro (média: 36,7 h/exame/paciente) neste estudo.

Em 94,5\% dos exames, não foram observadas complicações durante a utilização do CGMS. Não houve registro de trauma, infecção local ou sangramento em nenhum caso. Não houve nenhum caso de aversão psicológica, inoperabilidade técnica ou abandono do método, independentemente do tempo de CGMS.

\section{DISCUSSÃo}

O estudo realizado buscou demonstrar a correlação entre o tempo de hipoglicemia silenciosa pelo CGMS e o bom controle glicêmico (Alc versus média glicêmica) em pacientes com DMl. Verificou-se alto índice de hipoglicemia silenciosa captadas pelo sensor CGMS (65\% dos pacientes), predominantemente noturna. Quanto maior foi o tempo de hipoglicemia silenciosa menor foi a média glicêmica detectada.

Quanto à eficácia do sensor CGMS na identificação de excursões glicêmicas, observou-se alta eficácia do CGMS em relação à GC (4 vezes ao dia) $(\mathrm{p}<0,01)$, já comprovado pela literatura, inclusive em idosos institucionalizados $(4,6,15)$. A HPP foi identificada em número significativo de pacientes neste estudo $(60 \%)$.

A hipoglicemia silenciosa se mostra comum pela literatura, corroborado pelos dados apresentados, em cerca de $60 \%$ a $70 \%$ dos pacientes com DMl, principalmente no período noturno $(4-7,9,12,13,16,17)$. Dados recentes de Weber e cols. (18) evidenciaram alta freqüência de hipoglicemia silenciosa em pacientes DM2 submetidos à CGM. Guillod e cols. (19) verificaram hipoglicemia noturna em $67 \%$ de 88 pacientes com DMl, com melhora após seis meses da realização do CGMS. Boland e cols. (8) comparam os efeitos do CGMS versus GC em 56 crianças com DMl, por 72 horas. Observou-se superioridade do CGMS na detecção de excursões glicêmicas e de hipoglicemia assintomática (70\%), corroborado por Maia e Araújo (6). Observou-se maior tempo de hipoglicemia silenciosa durante a noite, de maneira significante, como encontrado por Jeha e cols. (20), em crianças DMl, por três dias. O tempo de hiperglicemia diurna elevado (metade do registro do CGMS em média) pode justificar os níveis médios de Alc e a média glicêmica nesse grupo, que pode ser mascarado pela presença da hipoglicemia noturna silenciosa.

Neste estudo, todos os pacientes foram orientados a manter a GC dentro dos horários já usuais, no mínimo, 4 vezes ao dia. Foram orientados quanto à medida noturna da glicemia, que não foi obrigatória. Em estu-

Tabela 2. Média glicêmica e Alc versus tempo de hipoglicemia silenciosa (\%) de pacientes portadores de DM1, submetidos à monitorização contínua de glicose por 72 horas.

\begin{tabular}{|c|c|c|c|c|c|}
\hline Variáveis & G1 (<5\%) & G2 (5\% a $10 \%)$ & G3 (10\% a $20 \%)$ & G4 (> 20\%) & $p$ \\
\hline & $n=36$ & $n=19$ & $\mathrm{n}=\mathbf{2 0}$ & $n=12$ & \\
\hline GMS (mg/dl) & $202,3 \pm 45,6$ & $186,4 \pm 45,2$ & $144,4 \pm 26,6$ & $127,83 \pm 25,7$ & 0,006 \\
\hline Alc (\%) & $9,4 \pm 1,7$ & $8,6 \pm 1,4$ & $8,4 \pm 1,8$ & $9,3 \pm 2,4$ & \\
\hline p valor & 0,001 & 0,01 & 0,04 & NS & \\
\hline
\end{tabular}

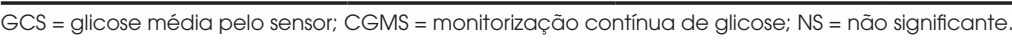


do recente no Brasil (SP), a calibração da madrugada, em 30 pacientes com $\mathrm{DMl}$, não interferiu na acurácia do CGMS ( $\mathrm{p}<0,0001$ ), não sendo recomendada de rotina no que se refere à eficácia do método (21). O pequeno número de mensurações da madrugada neste estudo (uma vez que não foi obrigatória) não possibilitou análise desses dados.

Durante mudanças rápidas e bruscas da glicemia se estima menor eficácia do método CGMS, em virtude do tempo de 5 a 10 minutos para transmissão dos dados do sensor ao monitor. Uma análise recente de $\mathbf{5 0}$ pacientes DMI (10 a 18 anos) utilizou freqüências variadas de GC de 3, 4, 6 e 7 vezes ao dia, em horários diversos, em períodos de maior e menor estabilidade glicêmica. Verificaram maior acurácia no grupo de maior inclusão de GC no monitor (7 vezes ao dia). No entanto, observaram que a freqüência de inclusão de GC no monitor parece ser menos importante que a realização e a sua inclusão em períodos de maior estabilidade glicêmica, antes de deitar e ao acordar (22).

As crises hipoglicêmicas são freqüentemente motivo de baixa insulinização intensiva em crianças e adolescentes com DMl, justificáveis por médicos e pacientes. Dados freqüentes da literatura estabelecem que a hipoglicemia leve a moderada não representa importante fator de déficit neurocognitivo motor em indivíduos diabéticos (23). Em cerca de metade das crianças com hipoglicemia não-diabética, com crises freqüentes de hipoglicemia grave, observou-se atraso de desenvolvimento leve e alterações discretas ao eletroencefalograma (24). Estima-se média de 0,2 eventos graves (coma hipoglicêmico ou crise convulsiva)/paciente/ano, não havendo recomendação para menor taxa de insulinização ou controle intensivo por causa do risco de complicações pela hipoglicemia em crianças e adolescentes com DMl (23).

O seguimento de quase 20 anos de pacientes do DCCT/EDIC ( $\mathrm{n}=1.439)$ verificou cerca de duas vezes mais eventos hipoglicêmicos no grupo de tratamento intensivo versus convencional, evidenciando ser a hipoglicemia complicação natural do controle glicêmico ideal. Os autores demonstraram não haver déficit neurocognitivo em pacientes com DMl, mesmo com história de hipoglicemias graves, ao longo de 18 anos de seguimento. No entanto, o controle glicêmico inadequado (Alc > 7\%) esteve associado a déficit psicomotor e velocidade motora $(\mathrm{p}<0,001)(25)$. Evidenciou-se, neste estudo, a correlação entre maior tempo de hipoglicemia silenciosa e menor média glicêmica em pacien- tes com $\mathrm{DMl}$, fato antes suposto pela literatura, mas desconhecido. Verificou-se tempo médio de $10 \%$ a $20 \%$ do registro de CGMS para se atingir média glicêmica satisfatória (120 a $160 \mathrm{mg} / \mathrm{dL}$ ), corroborando os dados recentes do DCCT/EDIC de que o maior prejuízo para o DMl parece ser a Alc/média glicêmica elevada e não o risco de hipoglicemias, uma vez que essa se mostra parte desse bom controle nesse grupo de pacientes (25).

A Alc representa o principal parâmetro de controle metabólico em pacientes com diabetes e representa a média glicêmica dos últimos três meses. Estima-se que cerca de $50 \%$ desse valor seja dado pelo último mês (30 dias), porquanto somente $10 \%$ é oriundo do controle prévio entre 90 a 120 dias, o que justifica modificações rápidas nos níveis de Alc após períodos curtos de ajuste de tratamento (14). Alguns fatores podem promover alterações dos resultados da Alc, como doenças genéticas e familiares hematológicas e técnica laboratorial inadequada. A beta talassemia minor não influenciou os resultados da Alc em indivíduos não-diabéticos (26). As presenças de hemoglobinopatias $\mathrm{C}$ e $\mathrm{S}$ também não estiveram relacionadas com alteração do método de análise da Alc, segundo a literatura (27).

De acordo com Kilpatrick e cols. (28), valores semelhantes de $\mathrm{Alc}$ em relação à média glicêmica podem ser influenciados pela população estudada (tratamento intensivo versus convencional). Ozmen e cols. (29) avaliaram 565 pacientes DM2 e verificaram alta correlação entre média glicêmica e Alc nos pacientes, tanto em jejum, pós-prandial e média de 7 pontos/dia ( $\mathrm{p}<$ $0,001)$. Detectaram sensibilidade de $84,5 \%$ e especificidade de $80,4 \%$ para média glicêmica de $175 \mathrm{mg} / \mathrm{dL}$, como parâmetro de bom controle metabólico $(\mathrm{Alc}<$ 7\%) (29). Neste estudo, verificou-se média glicêmica de $168 \mathrm{mg} / \mathrm{dL}$ para Alc $<7 \%$, em alta correlação com os dados de Rohlfing e cols. (14).

De acordo com o grupo de Rohlfing, a avaliação dos pacientes do DCCT $(\mathrm{n}=1.439)$, submetidos à glicemia capilar 7 vezes ao dia com a medida de Alc, a correlação entre glicemia média e Alc foi mais evidente no período pós-prandial e no noturno versus diurno/jejum. A medida da Alc foi realizada por HPLC, e uma estimativa de variação de $11 \%$ da glicemia capilar em relação à glicemia plasmática. Concluíram haver alta correlação entre média glicêmica e Alc em pacientes com DMl, com elevação de $35,6 \mathrm{mg} / \mathrm{dL}$ para cada $1 \%$ na Alc (Tabela 1 ). A variação intra-individual foi menor nos pacientes quando avaliados pela Alc $(9,7 \%)$ versus média glicêmica $(29,8 \%)$ 
(14). Este estudo demonstrou ser a média glicêmica de 7 pontos, usada por 18 vezes por paciente, parâmetro importante de controle glicêmico (estimativa de Alc). Em outra análise parcial de dados do DCCT, observouse que a média glicêmica e a variabilidade biológica (BV) exercem maior influência na Alc em relação à instabilidade glicêmica. A média glicêmica de dois a três meses foi considerada forte preditor de Alc. A instabilidade glicêmica só determinou $1 / 14$ do impacto na média glicêmica e $1 / 10$ na $\mathrm{BV}(30)$. $\mathrm{Na}$ análise dos dados apresentados, verificou-se maior nível de Alc no G4, mesmo na presença de menor média glicêmica, o que pode ter sido conseqüência do mau controle prévio ou maior tempo de hiperglicemia diurna, não diferenciada entre os grupos.

Segundo os dados de Kilpatrick e cols. (31), corroborados por Bolli (32), dos 1.441 pacientes do DCCT submetidos à GC de 7 a 8 vezes ao dia, concomitante à medida da Alc, a variabilidade glicêmica e os picos pré e pós-prandiais não foram preditores de complicações microvasculares (retino e nefropatias) em pacientes com DMl, sendo a média glicêmica o fator mais importante neste estudo $(31,32)$.

Portanto, este estudo mostrou a presença marcante da hipoglicemia silenciosa em pacientes com $\mathrm{DMl}$, predominante no período noturno, associada à média glicêmica tanto menor quanto maior o tempo de hipoglicemia silenciosa. Verifica-se a importância de abordar os pacientes em relação aos elevados níveis de Alc e média glicêmica versus hipoglicemia silenciosa, uma vez que essa parece ser parte integrante desse bom controle (Tabela 2). A segurança do baixo índice de efeitos neuropsicomotores pela hipoglicemia, ao longo de 20 anos evidenciados pelo DCCT/EDIC, reforça a importância de combater os altos níveis de Alc/média glicêmica em detrimento à preocupação com a hipoglicemia silenciosa, como barreira para insulinização mais intensiva.

Este trabalho representa a prática clínica real com essa nova terapia, com a percepção de que a hipoglicemia silenciosa é comum, ocorre mais à noite e é parte do bom controle desses pacientes, sendo a média glicêmica/Alc fora das metas possivelmente mais prejudicial aos pacientes, como verificado no estudo EDIC.

\section{CONCLUSÕES}

A hipoglicemia silenciosa é freqüente, mais duradoura à noite, e inversamente relacionada com a média glicêmi- ca. Verificou-se tempo médio de hipoglicemia silenciosa de $10 \%$ a $20 \%$ do registro de CGMS para se atingir média glicêmica satisfatória (120 a $160 \mathrm{mg} / \mathrm{dL}$ ), fato de enorme importância para o manejo do paciente $\mathrm{DMl}$, orientação dos pacientes e dos familiares, na busca de atingir as metas ideais e menor taxa de complicações em longo prazo.

Agradecimentos: À Roche Diagnóstica Ltda., pela concessão das fitas de glicemias usadas durante o estudo. Conflito de interesse: Dr. Levimar R. Araújo é membro consultor (board advisory) da Sanofi-Aventis, Eli-Lilly e Pfizer e atua com speaker das empresas Schering Plough e Medtronic.

\section{REFERÊNCIAS}

1. American Diabetes Association. Standards of Medical Care in Diabetes - 2007. Diabetes Care. 2007;30 Suppl 1:S4-41.

2. Moreira Jr ED, Mendes AB, Chacra AR. Avaliação do grau de controle glicêmico dos diabéticos no Brasil: resultados do estudo nacional da epidemiologia do diabetes. Arq Bras Endocrinol Metab. 2007;51 Suppl 1:S459.

3. The Diabetes Control and Complications Trial Research Group (DCCT). The effect of intensive treatment of diabetes on the development and progression of long term complications in insulindependent diabetes mellitus. N Engl J Med. 1993;329:977-86.

4. Maia FFR, Araújo LR. Efficacy of continuous glucose monitoring system (CGMS) to detect postprandial hyperglycemia and unrecognized hypoglycemia in type 1 diabetic patients. Diabetes Res Clin Pract. 2006;75(1):30-4.

5. The Diabetes Research in Children Network (DirecNet) Study Group: accuracy of the glucowatch G2 biographer and the continuous glucose monitoring system during hypoglycemia. Experience of the diabetes research in children network. Diabetes Care. 2004;27:722-6.

6. Maia FFR, Araújo LR. Accuracy, utility and complications of continuous glucose monitoring system (CGMS) in pediatric patients with type 1 diabetes. J Pediatr. 2005;81:293-7.

7. Kaufman FR. Role of continuous glucose monitoring in pediatric patients. Diabetes Technol Ther. 2000;2:S49-52.

8. Boland EA, Tamborlane VW. Continuous glucose monitoring in youth with type 2 diabetes: overcoming barriers to successful treatment. Diabetes Technol Ther. 2000;2:S53-9.

9. Maia FFR, Araújo LR. Impacto do sistema de monitorização contínua da glicose em pacientes diabéticos. Rev Assoc Med Bras. 2006;52(6):395-400.

10. Aussedat B, Dupire-Angel M, Gifford R, et al. Interstitial glucose concentration and glycemia: implications for continuous subcutaneous glucose monitoring. Arq Bras Endocrinol Metab. 2000;278:E716-28.

11. Kovatchev BP, Gonder-Frederick LA, Cox DJ, Clarke WL. Evaluating the accuracy of continuous glucose-monitoring sensors. Diabetes Care. 2004;27:1922-8. 
12. McGowan K, Tomas W, Moran A. Spurius reporting of nocturnal hypoglycemia by CGMS in patients with thigthtyle controlled diabetes. Diabetes Care. 2002;25:1499-503.

13. Ludvigsson J, Hanas R. Continuous subcutaneous glucose monitoring improved metabolic control in pediatric patients with type 1 diabetes: a controlled crossover study. Pediatrics. 2003;111:933-8.

14. Rohlfing CL, Wiedmeyer HM, Little RR, et al. Defining the relationship between plasma glucose and $\mathrm{HbA} 1 \mathrm{c}$. Diabetes Care. 2002;25(2):275-8.

15. Jamali R, Bachrach-Lindström M, Mohseni S. Continuous glucose monitoring system signals the occurrence of marked postprandial hyperglycemia in the elderly. Diabetes Technol Ther. 2005;7:509-15.

16. Yates K, Hasnat Milton A, Dear K, Ambler G. Continuous glucose monitoring-guided insulin adjustment in children and adolescents on near-physiological insulin regimens: a randomized controlled trial. Diabetes Care. 2006;29:1512-7.

17. Lagarde WH, Barrows FP, Davenport ML, Kang M, Guess HA, Calikoglu AS. Continuous subcutaneous glucose monitoring in children with type 1 diabetes mellitus: a single-blind, randomized, controlled trial. Pediatr Diabetes. 2006;7:159-64.

18. Weber KK, Lohmann T, Busch K, et al. High frequency of unrecognized hypoglycaemias in patients with type 2 diabetes is discovered by continuous glucose monitoring. Exp Clin Endocrinol Diabetes. 2007;115:491-4.

19. Guillod L, Comte-Perret S, Monbaron D, Gaillard RC, Ruiz J. Nocturnal hypoglycaemias in type 1 diabetic patients: what can we learn with continuous glucose monitoring? Diabetes Metab. 2007;23. [In press].

20. Jeha GS, Karaviti PL, Anderson B, et al. Continuous glucose monitoring and the reality metabolic control in preschool children with type 1 diabetes. Diabetes Care. 2004;27:2881-6.

21. Souza AGP, Akerman A, Perazo MN, Nery M, Melo KFS. CGMS: calibração da madrugada não interfere na acurácia do método. Arq Bras Endocrinol Metab. 2007;51(7):Suppl 1:S518.

22. Diabetes Research in Children Network (DirecNet). Evaluation of factors affecting CGMS calibration. Diabetes Technol Ther. 2006;8:318-25.
23. Jones TW, Davis EA. Hypoglycemia in children with type 1 diabetes: current issues and controversies. Pediatr Diabetes. 2003;4(3):143-150

24. Al Nassar S, Sakatai N, Al Ashwal A, Bin-Abbas B. Persistent hyperinsulinaemic hypoglycemia of infancy in 43 children: long-term clinical and surgical follow up. Asian J Surg. 2006;29(3):207-211.

25. DCCT/EDIC Study Group. Long-term effects of diabetes and its treatment on cognitive function. N Engl J Med. 2007;356:1842-52.

26. Polage C, Little RR, Rohlfing CL, Cole TG, Roberts WL. Effects of beta thalassemia minor on results of six glycated hemoglobin methods. Clin Chim Acta. 2004;350(1-2):123-8.

27. Roberts WL, Barun KSS, Rohlfing CL, et al. Effects of hemoglobin $\mathrm{C}$ and $\mathrm{S}$ traits on glycohemoglobin measurements by eleven methods. Clin Chem. 2005;51(4):776-8.

28. Kilpatrick ES, Rigby AS, Atkin SL. Variability in the relationship between mean plasma glucose and $\mathrm{HbA} 1 \mathrm{c}$ : implications for the assessment of glycemic control. Clin Chem. 2007;53(5):897-901.

29. Ozmen S, Cil T, Atay AE, et al. A simple way to estimate mean plasma glucose and to identify type 2 diabetic subjects with poor glycaemic control when a standardized HbA1c assay is not available. Diabet Med. 2006;23(10):1151-4.

30. RJ McCarter, HM James, SA Chalew. Mean blood glucose and biological variation have greater influence on $\mathrm{HbA1c}$ levels than glucose instability. Diabetes Care. 2006;29:352-5.

31. Kilpatrick ES, Rigby AS, Atkin SL. The effect of glucose variability on the risk of microvascular complications in type 1 diabetes. Diabetes Care. 2006;29:1486-90.

32. Bolli GB. Glucose variability and complications. Diabetes Care. 2006;29:1707-9.

\section{Endereço para correspondência:}

Frederico Fernandes Ribeiro Maia

Rua Nunes Vieira, 299, apto.702 - Santo Antônio

30350-120 Belo Horizonte, MG

E-mail: fredfrm@hotmail.com/frederico@diabetes.med.br 